Radiologe 2010 · 50:499

DOI 10.1007/s00117-010-2023-8

Online publiziert: 30 . April 2010

๑) Springer-Verlag 2010
T. Johnson · M.F. Reiser

Institut für Klinische Radiologie, Klinikum der

Ludwig-Maximilians-Universität München, Campus Großhadern, München

\title{
Moderne Herzdiagnostik mit CT und MRT
}

Sehr geehrte Kolleginnen und Kollegen,

nach wie vor führen kardiovaskuläre Erkrankungen die Todesursachenstatistik an. Daran hat die koronare Herzerkrankung einen erheblichen Anteil, und neben der Mortalität hat auch die Morbidität durch die ischämische Kardiomyopathie und Herzinsuffizienz eine wesentliche Bedeutung für die Lebensqualität der Bevölkerung und die Kosten im Gesundheitswesen. Von den valvulären Erkrankungen spielt vor allem die Aortenklappenstenose aufgrund der mit der Lebenserwartung steigenden Inzidenz eine zunehmende Rolle. In der Diagnostik stellt das Herz durch seine mehr oder weniger regelmäBige und nur in engen Grenzen beeinflussbare Bewegung und seine komplexe Anatomie große Herausforderungen an die nichtinvasive Diagnostik. In den letzten Jahren wurden durch die zunehmende räumliche und zeitliche Auflösung in der Schnittbildgebung wesentliche Fortschritte erzielt. CT und MRT haben in der Diagnostik des Herzens den Bereich der wissenschaftlichen Erprobung verlassen und vermögen in vielen Fällen wichtige, z. T. entscheidende Informationen beizutragen und können die Therapieentscheidungen maßgeblich bestimmen. Die Radiologen müssen nicht nur die Durchführung dieser anspruchsvollen Methoden beherrschen, sondern auch Kompetenz in der Indikationsstellung und der klinischen Bewertung der Ergebnisse erwerben, auch um den kardiologischen Kollegen dieses Potenzial bewusst zu machen und so dafür zu sorgen, dass es den Patienten zugute kommt.

In der Computertomographie hat die CT-Angiographie der Koronararterien ein Rennen um zusätzliche Schichten und v. a. eine höhere zeitliche Auflösung in Gang gesetzt - heute ist die Methode robust und als Routineanwendung etabliert. Zunächst wurde dieses Rennen ohne ausreichende Rücksicht auf die Strahlendosis geführt, was in den letzten Jahren zu einer berechtigten fachlichen und auch öffentlichen Diskussion um das Krebsrisiko geführt hat und den Fokus der technischen Entwicklung auf die Dosisreduktion lenkte. Arnoldi et al. stellen in dieser Ausgabe verschiedene aktuelle Niedrigdosistechniken im Detail dar. Eine Herausforderung bleiben Patienten mit koronarer Herzerkrankung, die bereits therapiert wurden. Während sich Bypassgefäße relativ gut darstellen lassen, sind Stents nur zum Teil in der CT beurteilbar. Diesem Thema widmen Seifarth et al. einen umfassenden Übersichtsartikel.

Auch in der MRT haben die Anforderungen der Herzbildgebung technische Entwicklungen bei der Anstiegssteilheit der Gradienten, bei Mehrkanalspulen und v. a. bei Sequenztechniken in Gang gesetzt. Abgesehen von der Angiographie der Koronararterien, die die klare Domäne der CT ist, hat sich die MRT als Goldstandard zur Diagnostik vieler relevanter Parameter durchgesetzt. Dazu zählen die globale Funktion des linken und rechten Ventrikels sowie die Vitalität des Myokards, und auch bei der Diagnostik der Wandbewegungsstörungen besitzt die MRT durch die Untersucherunabhängigkeit einen hohen Stellenwert. $\mathrm{Zu}$ den wichtigen Themen Funktionsauswertung, Vitalitätsbildgebung und Wandbewegung geben die Autoren Sandner et al., Bauner et al. und Kramer et al. einen Überblick über etablierte Methoden und neue Ent- wicklungen. Neben der KHK-Diagnostik bietet die MRT auch bei Klappenerkrankungen mit der Möglichkeit der morphologischen und dynamischen Darstellung von Herz und Gefäßen, mit Flussmessungen und Planimetrie von Öffnungsflächen, umfassende diagnostische Werkzeuge, die Beer et al. in einer Übersichtsarbeit darstellen.

Wir hoffen, dass dieses Themenheft mit der Kombination von Übersichts- und Originalarbeiten einen umfassenden und verständlichen Überblick über den Stand und aktuelle Entwicklungen der Herzbildgebung gibt und vielleicht bei einigen Kollegen das Interesse an diesem anspruchsvollen Gebiet weckt. Wir wünschen viel Spaß beim Lesen.

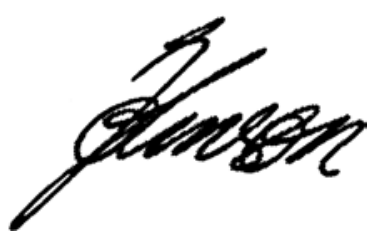

PD Dr. Thorsten Johnson

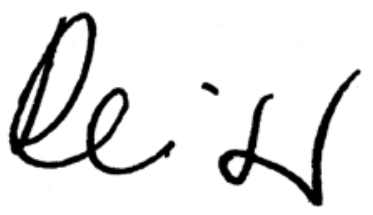

Prof. Dr. Dr. h.c. Maximilian F. Reiser 